\title{
Observations of the
}

Magnetosheath-Solar Wind Boundary

\author{
L. F. Burlaga $\%$ \\ and \\ K. W. Ogilvie
}

Laboratory for Space Sciences NASA-Goddard Space Flight Center Greenbelt, Maryland

April 1968

*NAS-NRC Postdoctoral Resident Research $\Lambda$ ssociate 


\section{Abstract}

Explorer 34 plasma data are presented which provide tests of current theories of the nature of the boundary between the earth's magnetosheath and the interplanetary medium. It is shown that a discontinuous transition between the magnetosheath and the interplanetary medium is seen simultaneously in the proton spectrum, the flow direction, and the magnetic field intensity, during both geomagnetically quiet and disturbed times. A few boundary crossings were observed for which the plasma spectra appear to indicate a diffuse transition; but a detailed study of an extreme example of such a crossing, based on simultaneous measurements of the spectra, the flow direction, and the magnetic field intensity, shows that the apparent diffuseness is actually the result of a complex microstructure which consists of a number of closely spaced discontinuities that could be due to multiple crossings of a bow shock. Observations of the velocity field in the neighborhood of the outer boundary of the magnetosheath are presented and are compared with results of the fluid theory, which predicts a standing shock. It is shown that,along the boundary on the flank of the magnetosheath, the ratios of the magnetosheath flow speed to the interplanetary flow speed are in close quantitative agreement with a prediction of the fluid theory. This prediction is essentially independent of the Mach number and the adiabatic exponent. We observe a highly dinectional flow in the region $30^{\circ} \leq \emptyset \leq 100^{\circ}$, where $\emptyset$ is the sun-earth-satellite angle. The direction

of this flow is in good agreement with the bow shock theory. 


\section{Introduction}

Axford (1962) predicted that a standing shock wave should exist permanently on the sunward side of the earth as a result of the flow of the supersonic interplanetary plasma past the earth's magnetosphere. Kellogg (1962) independently proposed the same idea and calculated the shape of the shock boundary using classical aerodynamic theory. for supersonic flow past an obstacle. More refined calculations of the shock shape were later published by Spreiter and Jones (1963) who used the fluid theory with more realistic boundary conditions. An alternative theory for the interaction between the interplanetary plasma and the earth's magnetic field was subsequently published by Bernstein et al (1964), Fredericks et al (1965), and by Scarf et al (1965). This free-flow theory implies a gradual, broad, disordered transition between the magnetosphere and the interplanetary medium. Ness (1967) in his review of experimental work on the solar wind geomagnetic field interaction has noted that there is, at present, disagreement as to which of these two theories is applicable.

There are a number of observations which support the shock theory: Boundary thickness. The magnetic field data of Ness et al (1964) and Heppner et al (1967) consistently showed a thin boundary between the magnetosheath and the interplanetary medium. Heppner et al (1967) have determined that the thickness of the boundary seen in the magnetic field is less than $\approx 250 \mathrm{~km}$. Argo et al (1966) reported that they saw no evidence for a diffuse boundary in the plasma data from Vela 2. 
Boundary shape. Dryer and Heckman (1967) have reviewed both plasma and magnetic field observations of the position of the boundary and have found that the observations are consistent with the shock theory. Velocity field. Examining the flow near the boundary at about $60^{\circ}$ and $300^{\circ}$ solar ecliptic longitude and between $\pm 56^{\circ}$ ecliptic latitude, Argo et al (1967) found that for 13 crossings the ratio of the magnetosheath flow speed to the interplanetary flow speed agreed with the theory of Spreiter et al (1966) and Dryer et al (1966). They observed an ordered, directional flow, as the shock theory predicts.

Temperature ratios. Spreiter et al (1968) have noted that the observations of the temperature ratios reported by Argo et al (1967) agree with the shock theory。

Observational evidence against the shock theory and in support of the free-flow theory was presented by Wolfe et al (1966a, 1966b): Boundary thickness. Wolfe et al (1966b) reported that they observed a diffuse boundary for more than half of the first twenty-one orbits of IMP 1. They suggested that the solar wind may be only marginally capable of producing a detached bow shock and that the shock may not form during geomagnetically quiet periods when the solar wind flux and speed is low.

Velocity field. Using data from four satellites (two near the subsolar point and one far out on each flank of the magnetosheath, near the magnetopause), Wolfe et al (1966a) found no increase in the bulk speed as the plasma flows around the magnetosphere. This is inconsistent with the shock theory. 
Clearly, the evidence in support of the fluid theory is substantial and convincing. The evidence against the fluid theory concerns the boundary thickness and the flow velocity behind the boundary. In this paper we examine the problem of the boundary thickness, using simultaneous plasma and magnetic field data, and we examine the plasma velocity taking care to consider several of the variables involved. Our results provide additional support for the fluid theory. 


\section{Instrument, Orbit and Data Reduction}

Explorer 34, launched on 27 May 1967, carries a plasma experiment provided by the present authors. Since a. full technical description will be presented elsewhere, (Ogilvie, McIlwraith and Wilkerson, 1968) only a condensed version will be given here.

Instrument. The experiment makes observations of the spectrum of ions in the energy per unit charge range $310 \mathrm{eV}$ per charge to $5100 \mathrm{eV}$ per charge. Fifteen energy intervals of width $4.5 \%$ are sampled, and their center energies form a geometric series with a ratio of 1.24 . An observation consists of counting ions whose energies fall in the pass band, and whose trajectories within the solid angle, of the analyser during one satellite revolution. As the sensitive angular cone of the experiment crosses the anti-solar direction, the energy sampled is changed to the next in order. The experimental program is shown in Figure 1, where it will be seen that in the course of nine telemetry sequences or about three minutes, three spectra are taken. The first of these is the energy spectrum of protons. The second is the energy spectrum of doubly cherged helium ions and the third consists of 15 successive readings on the same proton energy 'step'; helium data will not be discussed in this paper.

The time resolution of the experiment is specified as follows: A single observation of the ions of one species at one energy per unit charge setting occupies 2.6 seconds, a complete spectrum of ions of a single species requires approxinately one minute to record and these are repeated every 3 minutes. This program continues 
without interruption. The ion to be studied, $\mathrm{H}^{+}$or $\mathrm{H}_{e}^{++}$, is selected unambiguously by setting the velocity selector interposed between the analyser and the detector.

As an example of the operation of this device, Figure 2 shows a typical solar wind proton spect,rum. The degree of separation achieved between the two ions may be expressed as the ratio of the number of counts with the instrument adjusted to observe ion $A$, per incident ion $B$, values of about $10^{-4}$ are typical. The background seen in Figure 2 is a function of the operation of the computer which compresses the data before transmission, and is not caused by the detector. It is completely explained and determined during the recording of each spectrum so that it may readily be substracted before data reduction; there has been no change in this rate during the first 78 days in orbit. The threshold indicated in this figure represents a level of three standard deviations above the background level. Counting rates excetding the threshold are regarded as significant.

The sensitive cone of the instrument, which is two degrees in azimuth and $\pm 9^{\circ}$ in a plane containing the spin axis, rotates with its axis of symmetry in the plane of the ecliptic. By recording the number of particles detected by the instrument in successive $16^{\text {ths }}$ of a revolution, a series of 16 numbers, $C_{i}$, is obtained. Counting starts when the optical aspect sensor, looking out into a cone of angle $1^{\circ}$ whose axis is normal to the face of the sector opposite to that occupied by the plasma detector, sees the sun. The 16 subtotal counts then describe the azimuthal distribution of flux recorded as 
a histogram of 16 bars. One of the telemetered quantities is the number of the angular sector from which the greatest subtotal count $C \underset{i}{\max }$ was recorded. As seen from $\mathrm{Figure} 5$, and taking into account the $15 \frac{1}{2}$ degree displacement of the sensitive cone of the instrument and the 4 degree aberration correction, cold plasma flowing radially

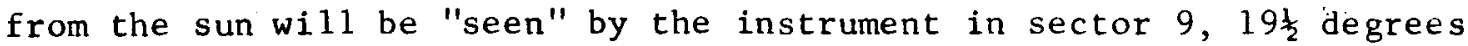
from the boundary between sectors 8 and 9 . Thus plasma flowing from a direction displaced by more than about 3 degrees westward of the sun will be detected in sector 10 , later in the rotation. A deflection of the flow direction towards the east by more than $19 \frac{1}{2}$ degrees will cause detection during the traversal of sector 8 . These qualitative angular estimates can be refined if the shape of the angular distribution is assumed; this point will not be pursued here.

Orbit. Figure 4 a shows the trajectory of Explorer 34 during orbit 2, and it shows the position of apogee on orbits 2 to 20 . It can be seen that the satellite was launched at a sun-earth-satellite angle $\emptyset_{\mathrm{SE}}=106^{\circ}$ and apogee moved at the rate of approximately $4.3^{\circ}$ per orbit toward the subsolar point, $\emptyset_{S E}$ decreasing. The orbital period is approximately 4.3 days. Apogee and perigee are $34 R_{E}$ and $<1 R_{E}$, respectively.

The orbital plane is nearly perpendicular to the ecliptic plane. Projections of the trajectory in the $\mathrm{X}, \mathrm{Z}$ plane are shown in Figure $4 \mathrm{~b}$ for several orbits; this is the projection which is seen when viewing away from the sun along the earth-sun line.

The spin axis of the satellite is oriented $2.5^{\circ}$ to the normal of the ecliptic plane; in what follows, we shall neglect this small angle. 
Reduction of Data to Obtain Fluid Parameters Details of the data reduction program are given in a report by ogilvie et a1.(1967). Briefly, the fluid parameters $n, u$ and $T$ are obtained from the observed spectra as follows (see Figure 2). 1) The working spectrum consisting of NOC adjacent bars is obtained by setting counts equal to zero in those energy channels in which the counts are less than some threshold. 2) The remaining counts are corrected for background and the counts in channel $i(i=L F, N O C-1)$ are then converted to a value of the distribution function $(\mathrm{dn} / \mathrm{dv})$ by means of the equation

$$
\left(\frac{\mathrm{dn}}{\mathrm{dv}}\right)=\text { constant } \times\left(\mathrm{C}_{i} / \mathrm{V}_{\mathrm{i}}^{2}\right)
$$

which is obtained from the solution of the unfolding problem that is given by Ogilvie et al. 3) The first three bars in the new working spectrum are fitted by the function

$$
f(v)=v \operatorname{vexp}\left[\alpha_{i}+\beta_{i} v+\gamma_{i} v^{2}\right]
$$

to obtain a representation to the actual observed distribution $f(v)=\frac{d n}{d v}(v)$ between channels $L F$ and $L F+1$; the next three bars $(L F+1, L F+2$, and $L F+3)$ are similarly fitted to obtain an approximaticn to $f(v)$ between $L F+1$ and $L F+2$, and this process continues until an analytical approximation to $f(v)$ is obtained by fitting all of the observed channels to segments of the function given by (1). 4) The fluid parameters are calculated by integrating the function $f_{a}(v)$ which is obtained by the fitting procedure: 


$$
\begin{aligned}
& n=\int_{0}^{\infty} f_{a}(v) d v \\
& <v>=\frac{1}{n} \int_{0}^{\infty} v f_{a}(v) d v \\
& T=\frac{m}{k n}\left\{\int_{0}^{\infty} v^{2} f_{a}(v) d v-n<v>^{2}\right\}
\end{aligned}
$$

This procedure gives reliable values of the fluid parameters in the interplanetary medium when the number of bars in the working spectrum is greater than or equal to three. In the magnetosheath, however, the parameters obtained in this way must be used with caution. The reason is that in the magnetosheath, where the temperature may be high and the bulk speed low, the angular spread of the distribution function may be greater than the aperature of the instrument. Consequently, the detector may not accept all of the particles, and the observed distribution $f_{a}(v)$ (which is an integral of $f(\vec{v})$ over a fraction of velocity space) may be significantly different from the actual speed distribution function $f(v)$ (which is an integral of $f(\vec{v})$ over all of the velocity space). This effect has been studied in detail for a convected maxwellian distribution. Equations (A 18) and (A 26) in the Appendix give the values of $\mathrm{dn} / \mathrm{dv}$ which should be observed for a given density, temperature, and bulk speed if the flow direction makes an angle $\theta_{0}$ with the axis of the acceptance cone of the detector which has a half-width $\Delta \theta \approx 9^{\circ}$. The $n_{0}, T_{0}$, and $u_{0}$ which shquld be observed for a given $n, T$, and $u$ have been computed from (3), (4), and (5) by setting $f_{a}=d n / d v$ where $d n / d v$ is given by (A 18) and (A 20). 
The results are shown in Figure 5. It can be seen that the mean speed $\left\langle v>\right.$ is essentially independent of $\theta_{0}$. The mean speed is generally higher than the flow speed $\mathrm{v}$, but this difference is less than $5 \%$ when the temperature is less than $5 \times 10^{5 \circ} \mathrm{K}$. The density is relatively insensitive to $\theta_{0}$ when $\mathrm{T} \approx 5 \times 10^{\circ} \mathrm{K}$, but it is more than a factor of two lower than the actual density when $\mathrm{T} \approx 10^{50} \mathrm{~K}$. Thus, in the magnetosheath we can set $\langle\mathrm{v}\rangle=\mathrm{u}$ when $\mathrm{T} \approx 10^{6{ }^{\circ}} \mathrm{K}$ with an error less than $10 \%$, but the errors in densicy may be large if no corrections are made. In the interplanetary medium the fluid parameters are correct to $\leqq 10 \%$ if $\mathrm{T} \lesssim 5 \times 10^{50} \mathrm{~K}$. 
III. Existence of a Discontinuous Transition Between the Magnetosheath and the Interplanetary Medium.

The bow shock theories which were mentioned in the Introduction imply that the boundary between the magnetosheath and the interplanetary medium should be very thin. Hydromagnetic shock theories such as that of Kennel and Sagdeev (1967) give a shock thickness somewhat larger than an ion cyclotron radius, viz. $\mathrm{L} \approx$ few $\times 100 \mathrm{~km} \approx .1 \mathrm{R}$. Shock theories involving ion wave instabilities, such as that of Tidman (1967), give a shock thickness on the order of the bulk-speed divided by the ion plasma frequency in the interplanetary medium, viz. $L \approx f e w \times 10 \mathrm{~km} \approx .01 \mathrm{R}_{\mathrm{E}}$. The magnetic field measurements of Heppner et a1. (1967) show that the major change during a bow shock crossing occurs within 30 seconds, from which they conclude that the thickness of the bow shock is less than $1 R_{E}$. Thus, although there is no agreement as to precisely what the shock thickness should be and although it is difficult to measure the thickness because of motions of the shock, proponents of any shock theory would probably agree that an upper limit to the apparent shock thickness is $\approx .1 \mathrm{R}_{\mathrm{E}}$. Since the Explorer 34 satellite typically moves approximately $1 \mathrm{R}_{\mathrm{E}}$ in several minutes near the outer bouniary, and since the plasma analyzer records successive proton spectra at three minute intervals, the shock theory implies that a shock crossing viewed on a scale of several hours would appear as a discontinuous transition between the interplanetary medium and the magnetosheath in the overwhelming majority of cases. On the other hand, the free flow theory apparently implies a very diffuse 
outer boundary with a scale on the order of a few earth radii (see Wolfe et a $1,1966 \mathrm{~b})$.

The purpose of this section is to show that we observe a discontinuous transition between the magnetosheath and interplanetary medium during both quiet and disturbed times. We shall show that this transition is seen simultaneously (within the three minute resolution of the plasma probe) by the magnetometer and the plasma probe. Quiet Time Transition. We shall first describe a transition between the magnetosheath and the interplanetary medium which was observed when the satellite moved from $17.4 \mathrm{R}_{\mathrm{E}}$ to $14.0 \mathrm{R}_{\mathrm{E}}$ at an earth-sun-apogee angle of $\sim 58^{\circ}$ in the interval $1600 \mathrm{UT}-1900 \mathrm{UT}$ on July 10, 1967. We wish to emphasize that July 10 was a very quiet day. Its IAGA designation is $\mathrm{QQ}$, the three-hour $\mathrm{Kp}$ indices for this day are $0^{+}, 0^{+}, 1,1^{-}, 0^{+}, 1,2$.

The lowest graph in Figure 6 shows the magnetic field intensity as a function of time. The low values of $B$ in the interval 1600 UT - 1804 UT are characteristic of the quiet time interplanetary magnetic fields, and the large magnetic field intensities in the interval 1804 UT - 1900 UT are characteristic of the outer magnetosheath (see Ness, 1967). The detailed magnetic field data show that the transition between these two regions occurred in less than 20 seconds. During this time the satellite mo:ed $.005 \mathrm{R}_{\mathrm{E}}$.

The plasma flow direction in the interval 1600 UT - 1900 UT on July 10 is shown above the magnetic field data in Figure 6. Prior to 1804 UT the flow is into channel 9 as one would expect from the nearly radial solar wind. After 1804 UT the flow is into channel 11. 
Section $V$ shows that such a flow direction is consistent with that predicted by the theory of Spreiter et al. (1966) for the region of the magnetosheath just behind the bow shock at $\emptyset_{\mathrm{SE}}=58^{\circ}$. Thus, the change in flow directions in Figure 6 indicates a discontinuity at 1804 UT. The width of the transition determined in this way is less than three minutes which corresponds to $L \leq .05 \mathrm{R}_{\mathrm{E}}$. Plasma spectral data for 1600-1900 UT on July 10 are summarized in the top graph of Figure 6 , which we refer to as a plasma spectrogram or "plasmagram". The vertical axis of the plasmagram shows speeds corresponding to the centers of the velocity channels of the plasma detector. Each column of numbers in the plasmagram shows a measure of the counts (see Table 1) which were recorded in the various energy channels during a one minute interval. In other words, each column represents a complete proton spectrum. The plasmagram is a series of these spectra ordered on a linear time scale. The narrow spectra in the interval 1600 UT - 1804 UT are characteristic of the interplanetary spectra which are observed during quiet times. The low bulk speed $(u \approx 300 \mathrm{~km} / \mathrm{sec})$ agrees with the quiet time interplanetary bulk speed reported by Wolfe et a1. (1966b) and by Hundhausen et a1. (1967) and also with theoretical predictions. The low temperature $\left(\mathrm{T} \geq 2 \times 10^{40} \mathrm{~K}\right)$ is also typical of the quiet interplanetary medium. The broad spectra after 1804 UT are undoubtedly magnetosheath spectra. We do not observe the low energy ends of these spertra because the peaks occurred near the lowest energy step of the instrument, so we cannot compute a mean speed or temperature. Nevertheless it is clear that the mean speed 
has decreased and the temperature increased considerably after 1804 UT. These spectra show a variability which is characteristic of magnetosheath spectra which we observed on other orbits.

In summary, the combined plasma and magnetic field data in Figure 6 show a discontinuous transition (boundary thickness $<.1 \mathrm{R}_{\mathrm{E}}$ ) between the interplanetary medium and the earth's magnetosheath during a geomagnetic quiet time. This agrees with the fluid theory. It is clearly incompatible with the free flow theory of the transition region. It is also inconsistent with the hypothesis that a discontinuous transition exists only at disturbed times. 
A "Diffuse" Transition During the first 15 orbits of Explorer 34 many discontinuous transitions were observed as the satellite moved between the interplanetary medium and the magnetosheath. However, there are a few cases $(\approx 5 \%)$ in which the plasma spectral data alone show a transition between the magnetosheath and the interplanetary medium which appears to be diffuse. An extreme and unusual example of such a transition is shown in the plasmagram in Figure 7.

In Figure 7 the broad, fluctuating spectra with low counts, which were observed during the interval 2110-220 UT, are characteristic magnetosheath spectra. The narrow spectra with higher counting rates, which were observed between 2230 and 2330 UT, are characteristic interplanetary spectra. There appears to be a gradual transition between these two types of spectra in the interval 2200-2230 UT。 During this time the satellite moved $.7 \mathrm{R}_{\mathrm{E}}$. This apparently diffuse boundary occurred during a geomagnetically quiet period in which the 3 hour $\mathrm{kp}$ index remained near 1 for 18 hours. Thus, in the absence of magnetic field data and without information about the plasma flow direction, it is easy to see how one might infer, with some justification, that a diffuse outer boundary might exist during a geomagnetic quiet period. However, we shall now show that more comprehensive data for this period indicate such a conclusion is probab1y incorrect. 
The magnetic field intensity shown in Figure 7 indicates that the satellite was in the magnetosheath between 2100 UT and 2217 UT。 At 2217 UT the magnetic field data show a discontinuous decrease to typical interplanetary intensities. This discontinuity was accompanied by a discontinuity in the flow direction from channel 11, which is typical of the magnet osheath, to channe1 10 , which is commonly observed in the interplanetary medium. A change in the character of the spectrum can also be observed near $1217 \mathrm{UT}$. The spectrum change alone is ambiguous, but the combination of observations just described definitely implies the crossing of a thin boundary. Another discontinuity, which is the complement of that just described, can be seen at 2323 UT. Here the magnetic field increases, the direction changes from sector 9 to 10 , and the spectrum broadens, indicating an abrupt transition from the interplanetary medium to the magnetosheath.

In the interval between 2231 UT and 2234 UT (marked M in Figure 7) the magnetic field data are characteristic of neither the magnetosheath nor the interplanetary medium. This observation is not inconsistent with the shock theory, for it is possible that the shock moved rapidly in and out during this interval so that both magnetosheath and interplanetary spectra were recorded but not clearly time resolved. Only one spectrum was recorded during this interval and the counts were too low to draw conclusions from it. Another "mixed" region can be seen between 2330 UT and 2335 UT in 
Figure 7. Here two spectra can be seen, but again the counts are too low to determine whether they indicate interplanetary or magnetosheath conditions. However, the magnetic field data strongly suggest that both conditions were observed in this interval as a result of multiple crossings.

Finally, let us consider the peculiar spectrum at 2358 UT. The spectrum is very broad which is indicative of the magnetosheath, but the flow is from channel 9, which is indicative of the interplanetary medium. This peculiar dilemma is readily resolved by referring to the magnetic field data. The magnetic field intensity shows that a discontinuous transition from the magnetosheath to the interplanetary medium occurred while the spectrum was being recorded. This implies that the spectrum should be a mixture of a magnetosheath spectrum and an interplanetary spectrum, consisting of low counts which were recorded in the low energy channels before the boundary crossing and higher counts which were recorded in the higher energy channels after the crossing. The observed spectrum does show these characteristics. Moreovis, the observation of flow from channel 9 is consistent with tis interpretation, for we plotted the flow direction which was recorded when the highest count was measured, and in this case the highest count was measured in the interplanetary part of the mixed spectrum.

We conclude that although the unusual plesmagram shown in Figure 7 may suggest, at first sight, a diffuse transition from 
the magnetosheath to the interplanetary medium, the apparent diffuseness actually is the result of a complex microstructure, which is revealed by simultaneously examining the plasmagram, the flow direction, and the magnetic field intensity. The microstructure consists of a series of discontinuities, which we interpret as multiple crossings of a thin boundary between the magnetosheath and the interplanetary medium. 
Typical Transitions On May 30, 1967, an unusual combination of "typical" bow shock crossings was observed in a six hour interval. This is shown in Figure 8 , which we shall now discuss in detail. At 1429 UT on May 30, 1967, a sudden commencement was observed at magnetic observatories around the world. At this time (to the resolution of our plasma analyzer), a marked discontinuity in the plasma and magnetic field parameters was observed by IMP $F$ which was near the bow shock, at $\mathrm{R} \approx 33.0 \mathrm{R}_{\mathrm{E}}, \emptyset_{\mathrm{e}} \approx 1009$ (see Figure 8 ). Prior to this time the geomagnetic field was quiet (for the first four 3-hour intervals on May 30 , the $\mathrm{Kp}$ indices are $2^{-}, 2,2^{+}$and $2^{-}$). After 1429 UT on May 30, the geomagnetic field was distrubed (for the last four 3-hour intervals on the date the $\mathrm{Kp}$ indices are $6^{-}, 6,6^{+}, 6^{-}$). Thus, Figure 8 shows data for both a quiet period and a disturbed period.

During the quiet period in Figure 8 , we see two types of regions. Between 1100 UT and 1308 UT, and between 1336-1406 UT, we observe a cool plasma with a low magnetic field moving nearly radially from the sun at $415 \mathrm{~km} / \mathrm{sec}$. This is clearly a "quiet" interplanetary plasma. Between 1308 UT at 1336 UT, and between 1406 UT and 1429 UT, the data in Figure 8 indicate that the satellite was in the quiet magnetosheath. Note that discontinuous transitions occurred simultaneously in the plasma and magnetic field data at $1308 \mathrm{UT}, 1336 \mathrm{UT}$, and $1406 \mathrm{UT}$. We assume that these are the result of motion of the bow shock back and forth past the satellite. We see such multiple crossings on nearly every orbit. 
During the disturbed period in Figure 8 (1429 UT - 1700 UT), we again see two types of regions. Between 1429 UT and 1557 UT, and between 1609 UT - 1700 UT, we see a hot, high speed plasma with a large magnetic field whose velocity fluctuates appreciably. This region is clearly the disturbed interplanetary medium. The other region, between 1557 UT and 1609 UT in Figure 8, is probably the disturbed magnetosheath. This is suggested by the very broad spectra, the flow direction (sector 11), the high magnetic field, and the low bulk speed. Note that discontinuous transitions between the disturbed magnetosheath and the distorted interplanetary medium occurred 1557 UT and at $1609 \mathrm{UT}$.

The above results lead us to the following interpretations of the events in Figure 8. Prior to 1409 UT, the satellite was near the bow shock, and the plasma near this region was rather quiescent. The bow shock was locally moving in and out, and twice it moved out past the satellite so that twice the instruments sampled the magnetosheath. Just before 1429 UT, the satellite was in the magnetosheath. At 1429 UT a discontinuity (presumably a shock wave which was propagating in the interplanetary medium) arrived at the satellite, and the disturbed plasma behind this discontinuity drove the bow shock past the satellite, toward the earth. Thus, at 1429 UT the satellite emerged from the quiet magnetosheath into a disturbed region in the interplanetary medium. The bow shock continued to move in and out. At 1557 UT it moved out past the satellite and it returned at 1609 UT, giving us a brief look at the disturbed magnetosheath. 
This event is unusual, but it also serves to illustrate the features of the outer boundary which are commonly observed in the plasma and magnetic field data, namely, multiple, discontinuous transitions between the interplanetary medium and the magnetosheath during both quiet and disturbed times. 
IV. Quiet-time Flow Speeds Near the Outer Magnetosphere

Boundary

Theory. Spreiter et al (1966) have used their steady state aerodynamic model of the earth's bow shock to compute $u_{s} / u_{i}$, $\mathrm{n}_{\mathrm{s}} / \mathrm{n}_{\mathbf{i}}$ and $\mathrm{T}_{\mathrm{s}} / \mathrm{T}_{\mathbf{i}}$ at positions along the bow shock for quiet. interplanetary conditions; here $u, n$ and $T$ are the flow speed, density and temperature, respectively, and the subscripts $s$ and $i$ refer to the magnetosheath and interplanetary medium, respectively. The authors show that these ratios depend only on the interplanetary free-stream Mach number $M_{\infty}$ and the adiabatic exponent $\gamma$ when the ratios are considered as functions of $X / D$, where $X$ is the distance measured from the earth toward the sun along the earth-sun line, and $D$ is given in units of earth-radii by the equation

$$
D=\frac{H_{p_{0}}{ }^{1 / 3}}{\left(2 \pi \rho_{i} u_{i}{ }^{2}\right)} 1 / 6
$$

where $\rho_{i}$ is the interplanetary proton density and $H_{\rho_{0}}$ is the earth's mean permanent magnetic $f i e l d$ at the geomagnetic equator $\left(\mathrm{H}_{\rho_{\mathrm{o}}} \approx .312\right)$. It is found that the density and temperature ratios are rather sensitive to the values of $M_{\infty}$ and $\gamma$. Since $\gamma$ is essentially unknown and since $M_{\infty}$ varies with time, we shall not consider density and 
temperature in this report. On the other hand, it is found that the theoretical flow speed ratio is not sensitive to the values of $M_{\infty}$ and $\gamma$ in the region $X / D \leq 6$. Thus the speed ratio gives a means of testing the shock theory which does not require an exact knowledge of $Y$, and it gives essentially a single curve with which we may compare speed ratios that are observed during a variety of interplanetary conditions.

To compare the theoretical speeds with the observed speeds, it must be understood that the theory as presented by Spreiter et al (1966) gives the flow speed $u$ which is essentially the speed with which the distribution function is convected, whereas the measurements give the mean speed of the observed particles which depends on both the convection and the thermal motion of particles. Specifically, the flow velocity $\vec{u}$ is defined in terms of the complete, normalized distribution function by the equation

$$
\vec{u}=\int \vec{v} f(\vec{v}) d \vec{v}
$$

where $d \vec{v}$ indicates that the integration extends over all of velocity space. This definition of flow speed is introduced when the field equations are derived by taking moments of the Boltzman equation. The mean speed, on the other hand, is defined by the equation

$$
<v>=\int_{0}^{\infty} v f(v) d v,
$$


where $f(v)$ is the speed distribution which is obtained by integrating $f(\vec{v})$ over all directions.

To relate $|\vec{u}|$ to $\langle v\rangle$ requires an assumption about $f(\vec{v})$. Since the fluid theory assumes a steady, equilibrium flow, the proper assumption is that $f(\vec{v})$ should be maxwellian. Using cartesian coordinates, it is easy to verify that if the distribution function $f(\vec{v})$ is maxwellian in a frame moving with speed $u$, then the magnitude of the flow velocity calculated from (7) is equal to $u$, i.e., $|\vec{u}|=u$. In the Appendix it is shown that for a maxwellian distribution which is convected with speed u,

$$
f(v)=\frac{d n}{d v}=\sqrt{\frac{m}{2 k T}} n\left(\frac{v}{u}\right) e^{\frac{m}{2 k T}(v-u)^{2}}
$$

(see (A16). Substituting this into (7) we find that $\langle v\rangle$ is related to $u$ by the equation

$$
<v>\approx u\left\{1+\frac{1}{2}\left(\frac{w}{u}\right)^{2}+0\left[\left(\frac{w}{u}\right)^{4}\right]\right\}
$$

where

$$
w=\sqrt{\frac{2 k T}{m}}
$$

It is convenient to write(11) in the form

$$
w(\mathrm{~km} / \mathrm{sec})=13 \sqrt{\frac{T 0 R}{10^{4}}}
$$


Note that the most probable speed $v_{p}$, which is obtained by setting the derivative of $f(v)$ in (8) equal to zero, is equal to the mean speed to order $(w / u)^{2}$. Thus the mean speed, the most probable speed and the flow speed $u$ are equal when $w / u<<1$. For example, in the interplanetary medlum $T \approx 5 \times 10^{4}$ and $u \approx 400 \mathrm{~km} / \mathrm{sec}$, so $\frac{1}{2}\left(\frac{w}{u}\right)^{2} \approx .003$ and $\left\langle v>\approx v_{p} \approx|\vec{u}|\right.$. In the magnetosheath, the difference between $\langle v\rangle$ and $|\vec{u}|$ may be significant. Since the higher order terms in (9) depend on the magnetosheath temperature, which is highly variable and cannot be accurately determined directly, a simple comparison between experiment and theory is not possible when the higher order terms are significant. From Figure 15 of Spreiter et al (1966) it is apparent that the higher order terms will be large when the interplanetary temperature and Mach number are high. For the purpose of testing the shock theory, we shall consider only bow shock crossings for which $T_{i} \leq$ $5 \times 10^{40} \mathrm{~K}$. In this case, the theory predicts that $\mathrm{T}_{\mathrm{s}} \leqslant 10^{60_{\mathrm{K}}}$ in the region $\mathrm{X} / \mathrm{D} \leq .6$ and $\mathrm{T}_{\mathrm{s}} \leq 5 \times 10^{50} \mathrm{~K}$ in the region $\mathrm{X} / \mathrm{D} \leq-.5$. For these numbers, we find that the magnitude of the term $\frac{1}{2}\left(\frac{w}{u_{s}}\right)^{2}$ is less than .05 and consequent $1 \mathrm{y}<\mathrm{v}_{\mathrm{s}}>\approx \mathrm{u}_{\mathrm{s}}$.

To summarize, we have noted that the bow shock theory gives a single curve for the flow speed ratio in the region $X / D \leq .6$, which is not sensitive to the values of $M_{\infty}$ and assumptions concerning $\gamma$. We have shown that during "quiet times" $\left(T \leq 5 \times 10^{40} \mathrm{~K}, \mathrm{u} \leq 450 \mathrm{~km} / \mathrm{sec}\right)$ the flow speed can be determined 
with an accuracy of a few percent. Thus, quantitative agreement between the measured and predicted flow speed ratios in the region $X / D \leqq .6$ is a necessary condition for the validity of the shock theory. We shall now present plasma data from the first ten orbits of Explorer 34 which show that the theory does indeed meet this test. 
Observations The measurements of flow speed rations $u_{s} / u_{i}$ for bow shock crossings which we shall now discuss were obtained during the first ten orbits of Explorer 34 when the satellite moved from $\emptyset=106^{\circ}$ to $\emptyset \approx 60^{\circ}$. Many boundary crossings were observed, since multiple crossings occurred on nearly every orbit, but only a few of these crossings are suitable for the test described above. We select data according to the following criteria:

1) The temperature on the interplanetary side of the boundary must be less than $5 \times 10^{40} \mathrm{~K}$.

2) At least five identifiable spectra on each side of the boundary must be available. Thus, crossings which occurred within 15 minutes of one another are not considered.

3) The flow speed in the magnetosheath must be sufficiently high $\left(u_{s} \geq 270 \mathrm{~km} / \mathrm{sec}\right)$ that the peak of the distribution function can clearly be seen.

When these criteria are applied we are left with boundary crossings for the first ten orbits for which we can compute $u_{s} / u_{i}$ as a function of $X / D$. For each of these boundary crossings we have at least five values of $u$ on both sides of the boundary. Ne have computed the ratio $u_{s} / u_{i}$ for each crossing in two ways.

1). By averaging the five values of $u_{s}$ and $u_{i}$. This method suffers from the disadvantage that the plasma parameters may change during the fifteen minutes required to obtain five spectra. 
2). By using the values of $u_{s}$ and $u_{i}$ appropriate to two single spectra. In order to avoid problems with "mixed" spectra we have not used the two spectra between which the boundary crossing took place, but have employed the spectra preceding and following these. We have verified that both methods of computing $u_{s} / u_{1}$ give essentially the same result. This gives a check on the validity of the steady flow assumption.

The results are shown in Figure 9 where $u_{s} / u_{i}$ are plotted as a function of $X / D$, where $D$ is computed from $n_{i}$ and $u_{i}$ using (5). The error bars in Figure 9 are simply estimates which were obtained by equating $\Delta u$ to one-half the difference of the maximum and the minimum values of the five measurements of $u$ and calculating $\Delta\left(u_{s} / v_{i}\right)$ from the equation

$$
\Delta\left(\frac{u_{s}}{u i}\right)=\frac{u_{s}}{u_{i}}\left[\left(\frac{\Delta u_{s}}{u_{s}}\right)^{2}+\left(\frac{\Delta u_{i}}{u_{i}}\right)^{2}\right]^{\frac{1}{2}}
$$

The largest error bars indicate either a low $u_{s}$, in which case we have set $u_{s}$ equal to the most probable speed, or a fluctuating flow speed in the sheath and interplanetary medium.

The theoretical curve in Figure 9 is taken from Figure 14 of Spreiter et al. (1966). It is evident that the observed speed ratios are in quantitative agreement with the theory within the errors of measurement. The measured and observed speed ratios differ by less than $10 \%$. 


\section{Flow Directions Near the Outer Boundary.}

In this section the flow directions which were observed in the magnetosheath near the outer boundary in the interval $30^{\circ} \leq \emptyset \leq 100^{\circ}$ are compared with the corresponding flow directions predicted by the shock model of Spreiter et a1. (1966)

In Section II it was explained that the flow direction is measured by determining in which of $16,22.5^{\circ}$ sectors the maximum number of counts was recorded during one satellite rotation. The relation between the sector number and the actual direction with respect to the sun is shown in Figure 4.

The flow direction data which will be presented were selected according to the following criteria:

1) The data were obtained within .5 $\mathrm{R}_{\mathrm{E}}$ of a clearly defined boundary crossing which was within $2.5 \mathrm{R}_{\mathrm{E}}$ of the theoretical boundary for $M_{\infty}=8, \gamma=2$.

2) The flow direction determined for a given rotation is used if and only if the total number of counts recorded during that rotation is greater than 6550 . The choice of the number 6550 is based on a study of the onboard computer which determines the flow direction. This criterion eliminates much of the data in the magnetosheath, where the counting rate is low, but it guarantees that the data which are selected are aecurate.

Seven of the first 18 orbits cf Explorer 34 gave more thar. one measurement of the flow direction which satisfies the two criteria 
above. The measurements are summarized in Table 2. Accurate data were obtained on orbits $2,3,5,7,12,14$, and 18 . On each orbit the most probable flow direction was into sector 11 . However, a few observations of flow into sector 10 and sector 12 were recorded, this could indicate a small variability of the flow direction or a residual instrumental effect. It is useful to define a mean sector for each orbit by the equation

$$
\text { mean sector } \equiv \sum N_{i} S_{i}^{\prime} \sum N_{i}
$$

where $N_{i}$ is the number of observations of flow into sector $s_{i}$. For example, on orbit 2, 16 measurements of the flow direction were made which satisfied the selection criteria; 4 measurements indicated flow into sector 10 , and 12 measurements indicated flow into sector 11 , so the mean sector $1510.7 \pm .4$. Values of the mean sectors, given in Table 2, show that the flow was predominantly into channel 11; the RMS errors in the mean sectors shows that the flow was highly collimated.

The measured most probable, flow directions are compared with the theoretical flow directions of Spreiter et al (1966) in Figure 10. . This Figure shows the theoretical boundary and the theoretical streanlines for the model with $M_{\infty}=8, \gamma=2, D=10 R_{E}$ (Spreiter et al have shown that the streamlines for $M_{\infty}=8, \gamma=5 / 3$ and $D=10 R_{E}$ are essentially the same). The measured flow direction for each boundary crossing is shown in Figure 10 as flow into a narrow sector which is placed at the shock boundary at the appropriate $\emptyset$. It is evident that the predicted and measured flow directions are in good agreement. 
VI. Conclusions

We have presented plasma and magnetic field data from Explorer 34 which are relevant to the study of the nature of the boundary between the magnetos heath and the interplanetary medium. The results may be summarized as follows:

1) The boundary is characterized by a discontinuous change in the proton spectrum, the flow direction and the magnetic field intensity. The discontinuities in the plasma and magnetic field are simultaneous within the 3-minute resolution of the plasma analyzer. The thin boundary is observed during both geomagnetically quiet and disturbed times. There is no evidence for a diffuse boundary as suggested by the free flow theory.

2) The flow speed in the region $-.65 \leq \frac{X}{D} \leq .65$ agrees quantitatively (within $10 \%$ ) with a prediction of the shock theory of Spreiter et al (1966) which is essentially independent of the Mach number and the adiabatic exponent. 3) The measured flow direction in the region $30^{\circ} \lesssim \emptyset \lesssim 100^{\circ}$ agrees closely with the theory of Spreiter et al (1966). The flow is highly directional, as predicted.

Recall that other measurements which have been published plasma measurements of the position of the outer boundary (Dryer and Heckman, 1967), magnetic field measurements of the position of the boundary thickness (Heppner et al. 1967), and the plasma measurements of the change in density, bulk speed, and temperature across the boundary at $15 \mathrm{R}_{\mathrm{E}}$ (Argo et al., 1967) - are also in quantitative 
$-31-$

agreement with predictions of the shock theory. These observations together with the results of this paper provide strong, quantitative support for the fluid theory of the interaction of the solar wind with the earth's magnetosphere. 


\section{APPENDIX}

The purpose of this appendix is to present the convected maxwellian distribution function $f(\vec{v})$ in spherical coordinates, and to calculate the functional form of the distribution, $\mathrm{dn} / \mathrm{dv}$, which the IMP F plasma probe records in a convected maxwellian plasma.

In a coordinate system which moves with the plasma the maxwe11ian distribution has the form

$$
f(\vec{v})=\frac{d^{3} n}{d v_{x} d v_{y} d v_{z}}=A e^{-\alpha\left[v_{x}^{2}+v_{y}^{2}+v_{z}^{2}\right]}
$$

where

$$
\alpha=\frac{\mathrm{m}}{2 \mathrm{kT}}
$$

and

$$
A=n\left(\frac{m}{2 \pi k T}\right)^{3 / 2}
$$

Consider a fixed coordinate system such that the $v_{z}$ axis is parallel to the spin axis (approximately perpendicular to the ecliptic plane) and the bulk velocity is in the $v_{y}-v_{z}$ plane. In a spherical coordinate system the bulk velocity makes an angle $\theta_{0}$ with the ecliptic plane and a particle has a velocity given by $(v, \theta, \emptyset)$. The transformation equations which relate $\left(v_{x}, v_{y}, v_{z}\right)$ to $(v, \theta, \phi)$ are 


$$
\begin{aligned}
& v_{x}=v \cos \theta \sin \emptyset \\
& v_{y}=v \cos \theta \cos \emptyset-u \cos \theta_{0} \\
& v_{z}=v \sin \theta-u_{\sin } \theta_{0}
\end{aligned}
$$

It follows that

$$
v_{x}^{2}+v_{y}^{2}+v_{z}^{2}=v^{2}+u^{2}-2 u v \cos \theta \cos \emptyset_{\cos } \theta_{o}-2 u v \sin \theta \sin \theta_{o}
$$

and it can readily be shown that the Jacobian of the transformation is

$$
\frac{\partial\left(v_{x}, v_{y}, v_{z}\right)}{\partial(v, \theta, \emptyset)}=v^{2} \cos \theta
$$

Substituting $A 5$ and $A 6$ into $A 1$ gives the first result:

$$
\frac{\mathrm{dn}}{\mathrm{dvd} \theta \mathrm{d} \phi}=A v^{2} \cos \theta \mathrm{e}^{-\alpha\left[\mathrm{v}^{2}+\mathrm{u}^{2}-2 u \cos \theta_{\mathrm{O}} \mathrm{v} \cos \theta \cos \phi-2 u \sin \theta_{\mathrm{O}} v \sin \theta\right]}
$$

This is the equation for the distribution functicn of a maxwellian gas convected with speed $u$, expressed in spherical coordinates. The Explorer 34 plasma probe has a narrow aperature which admits only particles for which $-\Delta \theta \leq \theta \leq \Delta \theta$. It is sensitive only to the velocity component $v \cos \theta$; but $|\theta| \leq \Delta \theta \approx 9^{\circ}$ for the particles which are detected, so $\mathrm{v} \cos \theta \approx \mathrm{v}$. The probe integrates the velocity distribution over all $\emptyset$ by virtue of its spin about the $v_{z}$ axis, and it integrates over the interval $-\Delta \theta \leqslant \theta \leqslant \Delta \theta$ by 
virtue of its aperature in the $\theta$ direction. Thus, the instrument measures

$$
\frac{d n}{d v}=A v^{2} e^{-\alpha\left(v^{2}+v^{2}\right)} \times Q_{\theta}
$$

where

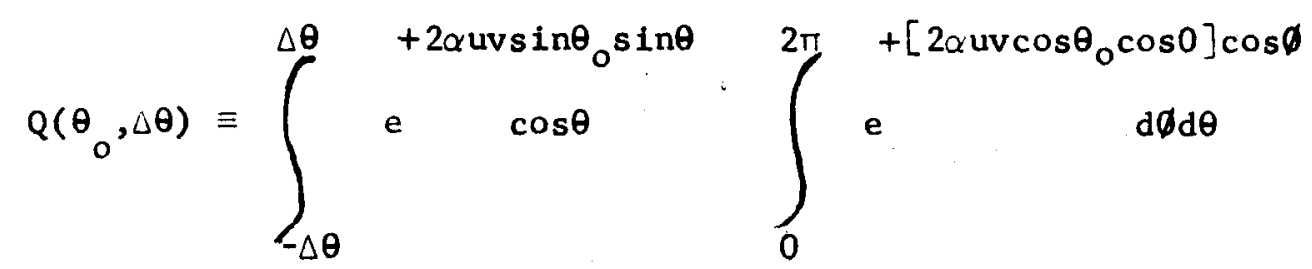

The $\emptyset$ integral in $Q\left(\theta_{0}, \Delta \theta\right)$ is equal to

$$
Q_{\emptyset}=2 \pi I_{0}\left(2 \alpha u v \cos \theta_{0} \cos \theta\right)
$$

where $I_{0}$ is the modified Bessel function of order zero.

Since $\alpha$ is on the order of the reciprocal of the thermal speed squared which is generally much less than uv, and since we are interested in $\operatorname{small} \theta_{0}$ and $\theta$, we can use the asymptotic expansion for the Bessel function to obtain

$$
Q_{\emptyset}=\frac{\sqrt{2 \pi} e^{2 \alpha u v \cos \theta_{0} \cos \theta}}{\sqrt{2 \alpha u v \cos \theta_{0} \cos \theta}}
$$


The remaining integral in (A8) is then

$$
Q_{\emptyset}=\frac{\sqrt{2 \pi}}{\sqrt{2 \alpha u v \cos \theta_{0}}} \times \int_{-\Delta \theta}^{\Delta \theta} e^{2 \alpha u v\left(\sin \theta_{o} \sin \theta+\cos \theta_{o} \cos \theta\right)} \frac{\sqrt{\cos \theta} d \theta}{d \theta}
$$

We are interested in small $\theta$ (since $\left.\Delta \theta \approx 9^{\circ}\right)$ and $\operatorname{small} \theta_{0}$, (since the flow is nearly normal to the spin axis of the detector), so we can expand the trigonometric functions in a Taylor's series to obtain

$$
Q_{\theta}=\frac{\sqrt{2 \pi}}{\sqrt{2 \alpha u v}} \int_{-\Delta \theta}^{\Delta \theta} e^{\left[2 \alpha u v\left(\theta \theta+1-\frac{\theta^{2}}{2}\right)\right]} d \theta
$$

Completing the square in the exponential and neglecting $\theta_{0}^{2}$ compared with 2 gives

$$
Q_{\theta}=\frac{\sqrt{2 \pi}}{\sqrt{2 \alpha u v}} e^{2 \alpha u v} \int_{-\Delta \theta}^{\Delta \theta} e^{-2 u v\left(\theta-\theta_{0}\right)^{2}} d \theta
$$

Note that this implies that the $\theta$ dependence of the distribution $f(\vec{v})$ is approximately Gaussian with a maximum at $\theta=\theta_{0}$. This is a common assumption (Argo et al. 1967). Now we shall distinguish two cases.

1. Interplanetary Medium. In the interplanetary medium essentially all of the particles move with $\left(\theta-\theta_{0}\right)$ small, of order 3 degrees, and $\theta_{0}$ is commonly of the same order, so we can extend the limits in (A14) to $\pm \infty$. 
In this case

$$
Q_{\theta}=\frac{\pi}{\alpha u v} e^{2 \alpha u v}
$$

Thus, we find from (A8) and (A14) that in the interplanetary medium

$$
\frac{\mathrm{dn}}{\mathrm{dv}}=\pi \frac{\mathrm{A}}{\alpha}\left(\frac{\mathrm{v}}{\mathrm{u}}\right) \mathrm{e}^{-\alpha(\mathrm{v}-\mathrm{u})^{2}}=\mathrm{n} \sqrt{\frac{\mathrm{m}}{2 \pi \mathrm{k}}} \frac{1}{\sqrt{\mathrm{T}}}\left(\frac{\mathrm{v}}{\mathrm{u}}\right) \mathrm{e}^{-\frac{\mathrm{m}}{2 \mathrm{kT}}(\mathrm{v}-\mathrm{u})^{2}}
$$

where

$$
\sqrt{\frac{m}{2 \pi k}}=.436 \times 10^{-4} \text { if cgs units are used. }
$$

2. Magnetosheath. If the flow speed is low and the temperature is high, as may occur in the magnetosheath, then one cannot extend the limits of the integral in (A13). However, the integral can be expressed in terms of error functions:

$$
\begin{gathered}
\text { a) If } \theta_{0}<\Delta \theta \\
Q_{\theta}=\frac{\pi}{2 \alpha u v} e^{2 \alpha u v}\left\{\operatorname{erf}\left[\sqrt{\alpha u v}\left(\theta_{0}+\Delta \theta\right)\right]+\operatorname{erf}\left[\sqrt{u u v}\left(\theta_{0}-\Delta \theta\right)\right]\right\}
\end{gathered}
$$

and we find that

$$
\begin{aligned}
& \frac{\mathrm{dn}}{\mathrm{dv}}=\frac{.436 \times 10^{-4}}{2} \frac{\mathrm{n}}{\sqrt{\mathrm{T}}} \frac{\mathrm{v}}{\mathrm{u}} \mathrm{e}^{-\frac{\mathrm{m}}{2 \mathrm{kT}}(y-u)^{2}} \vdots \operatorname{erf}\left[\sqrt{\frac{\mathrm{a}}{2}}\left(\theta_{\mathrm{o}}+\Delta \theta\right)\right]+ \\
& \left.\operatorname{erf}\left[\sqrt{-\frac{a}{2}}\left(\theta_{0}-\Delta \theta\right)\right]\right\}
\end{aligned}
$$


where

$$
a=\frac{m}{k} \frac{u^{2}}{T}\left(\frac{v}{u}\right)=1.21 \times 10^{-8} \frac{u^{2}}{T} \frac{v}{u}
$$

b) If $\theta_{0}>\Delta \theta$

then

$$
\frac{\mathrm{dn}}{\mathrm{dv}}=\frac{.436 \times 10^{-4}}{2} \frac{\mathrm{N}}{\sqrt{\mathrm{T}}} \mathrm{e}^{-\frac{\mathrm{m}}{2 \mathrm{kT}}}\left(\mathrm{v-u)^{2 }} \frac{\mathrm{v}}{\mathrm{u}}\left\{\operatorname{erf}\left[\sqrt{\frac{\mathrm{a}}{2}}\left(\theta_{\mathrm{o}}+\Delta \theta\right)\right]-\operatorname{erf}\left[\sqrt{\frac{\mathrm{a}}{2}}\left(\theta_{\mathrm{o}}-\Delta \theta\right)\right]\right\}\right.
$$

and $a$ is given by (A19). 
Acknowledgements

We wish to thank Drs. N. F. Ness and D. H. Fairfield for permitting us to use the Explorer 34 magnetic field data, and we acknowledge helpful conversations with them。 Professor T. D. Wilkerson made important contributions to this report in the form of many discussions about both the theory and the experiment. The data were reduced with the diligent and competent programming assistance of M. Courtney, D. Graves, and R. Ryder。 
Re ference

Argo, H. V., J. R. Asbridge, S. J. Bame, A. J. Hundhausen, and I. B.

Strong, Observations of Solar Wind Plasma Changes Across the Bow Shock, J. Geophys. Res., 72, 1989, 1967.

Axford, W. I., The Interaction between the Solar Wind and the Earth's Magnetosphere, J. Geophys. Res., 67, 3791, 1962.

Bernstein, W., R. W. Fredricks, and F. L. Scarf, A Model for a Broad Disordered Transition between the Solar Wind and the Magnetosphere, J. Geophys. Res., $\underline{69}, 1201,1964$.

Dryer, M., and G. R Heckman, On the Hypersonic Analogue as Applied to Planetary Interaction with the Solar Plasma, Planet. Space Sci., 15, 515, 1967.

Fredericks, R. W., F. L. Scarf, and W. Bernstein, Numerical Estimates of Superthermal Electron Production by Ion Acoustic Waves in the Transition Region, J. Geophys. Res., 70, 21, 1965.

Heppner, J. P., M. Sugiura, T. L. Skillman, B. G. Ledley, and M. Campbe11, OGO-A Magnetic Field Observations, I. Geophys. Res., 2ㅡ, 5417, 1967. Hundhausen, A. J., J. R. Asbridge, S. J. Bame, and J. B. Strong, Vela Sate11ite Observations of Solar Wind Ions, I. Geophys. Res., 72 , $1979,1967$.

Kellogg, P. J., Flow of Plasma Around the Earth, I. Geophys. Res., 67, 3508,1962 .

Kenne1, C. F., and R. Z. Sagdeev, Collisionless Shock waves in High $\beta$ Plasmas, 1 and 2, J. Geophys. Res., 72, 3303, 1967. 
Ness, N. F, Observations of the Interaction of the Solar Wind with the Geomagnetic Field during Quiet Conditions, Solar-Terrestrial Physics, Academic Press, Inc., (London) Limited, pp. 58-89, 1967. Ness, N. F., C. L. Scearce, and J. B. Seek, Initial Results of the IMP-1 Magnetic Field Experiment, J. Geophys. Res., 69, 3531, 1964.

Ogilvie, K. W., L, F. Burlaga, and H. Richardson, Analysis of Plasma Measurements on IMP-F, NASA Technical Rept, X-612-67-543, 1967.

Ogilvie, K. W., N. McIlwraith, and T, D. Wilkerson, A Mass-Energy Spectrometer for Space Plasmas, Rev. Sci. Instr. (in press), 1968. Scarf, F. L., W. Bernstein, and R. W. Fredericks, Electron Acceleration and Plasma Instabilities in the Transition Region, J. Geophys. Res., 70, 9,1965 .

Spreiter, J. R., and W. P. Jones, On the Effect of a Weak Interplanetary Magnetic Field on the Interaction between the Solar Wind and the Geomagnetic Field, J. Geophys. Res., 68, 3555, 1963.

Spreiter, J. R., A. L. Summers, and A. Y. Alksne, Hydromagnetic Flow Around the Magnetosphere, Planet. Space Sci., 14, 223, 1966.

Spreiter, J.R., A. L. Summers, and A. Y. Alksne, On the Comparison of Temperature Jumps across the Earth's Shock - Vela 3 Satellites and Gasdynamic Theory, J.Geophys. Res. 73, 1851, 1968.

Tidman, D. A., The Earth's Bow Shock Wave, ‥ Geophys. Res., 72, 1799, 1967.

Wolfe, J. H., R. W. Silva, and M. A. Myers, Preliminary Results from the Ames Research Center Plasma Probe Observations of the So:ar Wind on IMP II and CGO-I, Space $\underline{\text { Res. }}, \underline{6}, 680,1966 \mathrm{a}$.

Wolfe, J. H., R. W. Silva, and M. A. Myers, Observations of the Solar Wind during the Flight of Imp 1, J. Geophys. Res., 71, 1319, 1966b. 


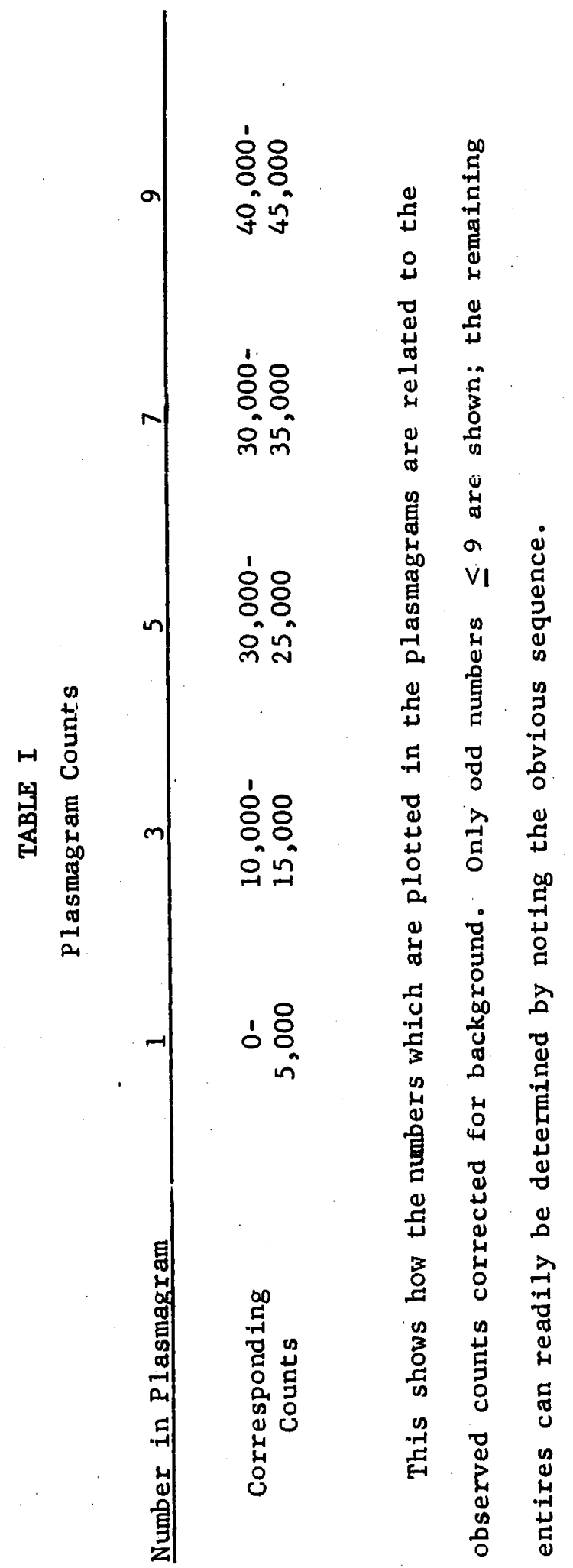




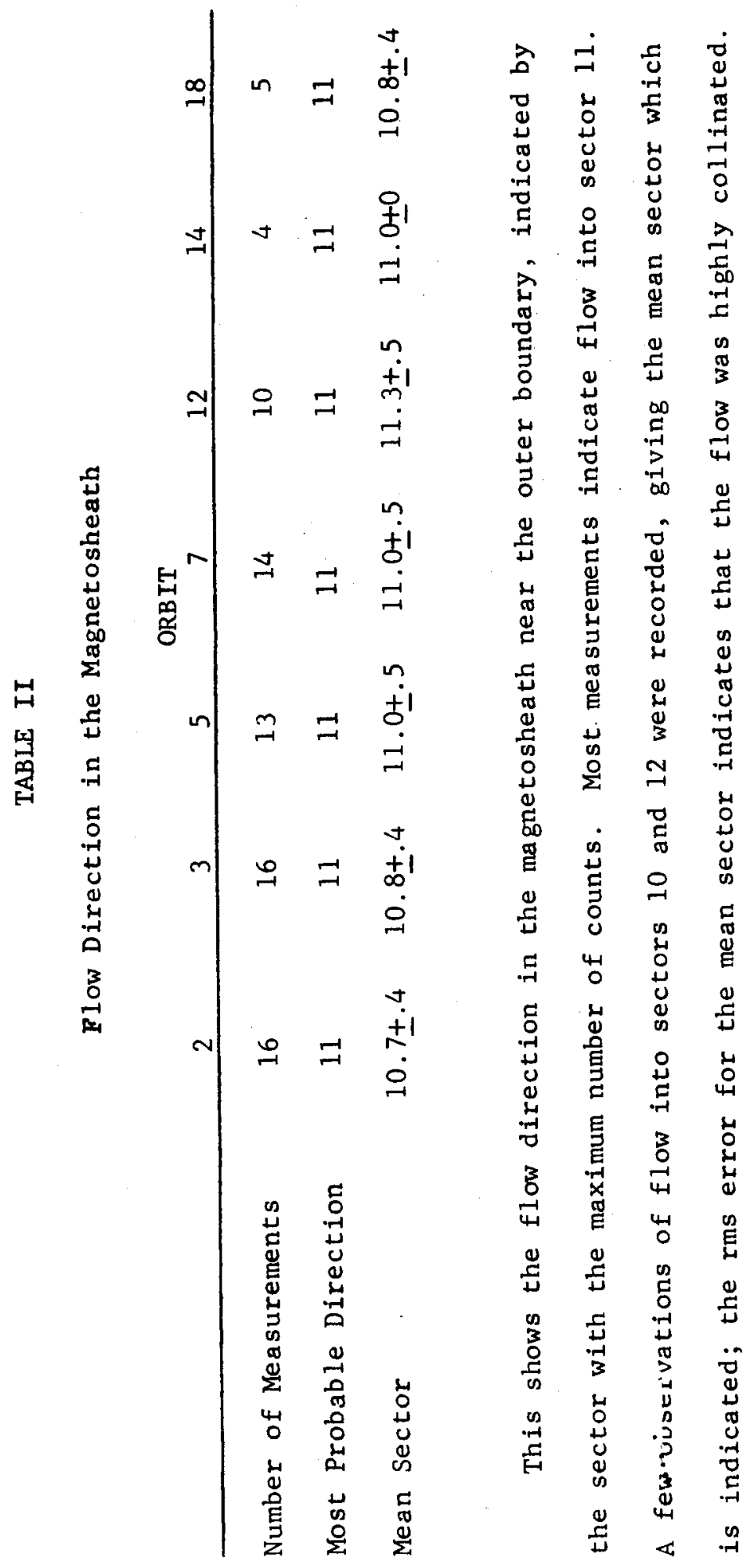




\section{Figure Captions}

Figure 1. This shows how the telemetry encoding takes place. Data for five spins, 1/3 of a spectrum, are transmitted during one sequence. The instrument cycles between the proton, helium, and fluctuation modes. Three cycles, approximately three minutes, are required to obtain a complete spectrum, which consists of 15 measurements.

Figure 2. This shows the definition of a working spectrum and illustrates the fitting procedure. The spectrum shown here (a disturbed magnetosheath spectrum) is not typical. It was chosen to demonstrate that a general fitting procedure is needed because the spectra are not all maxwellian. The dashed line between any two bars is obtained by fitting three bars to a convected maxwellian distribution; for example, the curve between bars 1 and 2 in the working spectrum is obtained by fitting bars 1,2 , and 3 .

Figure 3. The upper diagram shows the arrangement of the detector relative to the sun sensor in the satellite. The sensitive cone of the detector is $\approx 2^{\circ}$ wide. The rotation direction shown here is that which is seen by an observer south of the ecliptic plane. The lower diagram shows the sector pattern relative to the earth-sun line, which is seen by an observer north of the ecliptic plane. The on-board computer stores the counts which are recorded as the sensitive cone moves through each of these sectors; the counts are recorded in the sequence given by the numbering of the sectors. 
Figure 4. Figure 4 a shows the ecliptic plane projection of the orbit 2 trajectory of Explorer 34, and it shows the position of apogee for orbits 2 to 20. Figure 4b shows the $\mathrm{Y}-\mathrm{Z}$ projection (projection in a plane perpendicular to the earth-sun line) for orbits $2,6,10,14,18$ and 20 .

Figure 5. We have calculated the mean speed $\langle v\rangle$ and densitity $n_{0}$ which would be observed by the plasma detector if the actual distribution function is a convected maxwellian distribution with density $n$, temperature $T$, and both velocity $\vec{u}$. Here we show how $<v>/|\vec{u}|$ and $n_{0} / n$ vary as a function of $\theta_{0}$, where $\theta_{0}$ is the direction of $\vec{u}$ as shown in Figure 11 .

Figure 6. A plasmagram, the flow directions, and the magnetic field intensity are shown for a four-hour interval during a gepmagnetically quiet day (IAGA designation, QQ). A distinct, discontinuous boundary crossing can be seen at 1804 UT. There appears to be a very brief excursion into the magnetosheath at $1710 \mathrm{UT}$.

Figure 7. The plasmagram above seems to indicate a diffuse transition between the magnetosheath and the solar wind; but the flow direction and magnetic field intensity indicate that the apparent diffuseness is the result of a complex microstructure consisting of many discontinuities. The letters S, I, and M indicate magnetosheath, interplanetary medium, and "mixed", respectively. 
Figure 10. The solid curves show the theoretical streamlines and shock boundary computed by Spreiter et al. (1966) for $M_{\infty}=\delta$, $Y=2$; the streamlines for $Y=5 / 3$ are essentially the same. The observed flow direction was into the solid sectors which are shown. The sectors shown here have been moved slightly in each case so that they fall on the theoretical shock boundary. The data were selected so that the observed boundary and flow direction were in most cases within $1 R_{E}$ of the theoretical boundary.

Figure 11. This shows the coordinate system which is used for the calculations in the Appendix. 
Figure 8. An unusual combination of typical transitions between the magnetosheath and the solar wind. The discontinuity at 1409 UT was produced by an interplanetary shock wave that presumably drove the bow shock toward the earth past the satellite, which was in the magnetosheath just before 1409 UT. Prior to 1409 UT we see samples of the quiet magnetosheath and quiet solar wind; after 1409 UT we see samples of the disturbed magnetosheath and the disturbed solar wind. The multiple crossings shown here are commonly observed; we consider these to be the result of motions of a single thin boundary.

Figure 9. This shows the ratio of the measured mean speed on the magnetiosheath side of the boundary to the measured mean speed on the solar wind side of the boundary, plotted as a function of $X / D$, where $X$ is the coordinate of the satellite on the earth-sun 1ine, and $\mathrm{D}$ is the theoretical postion of the magnetopause boundary at the subsolar point. The data were selected so that the interplanetary temperature was $<5 \times 10^{40} \mathrm{~K}$; for these data the mean speed is essentially equal to the flow speed. The solid curve is the theoretical flow speed ratio given by spreiter et a1. (1966). The theoretical curve is essentially independent of the Mach number and the adiabatic exponent $\gamma$ in the interval shown. 


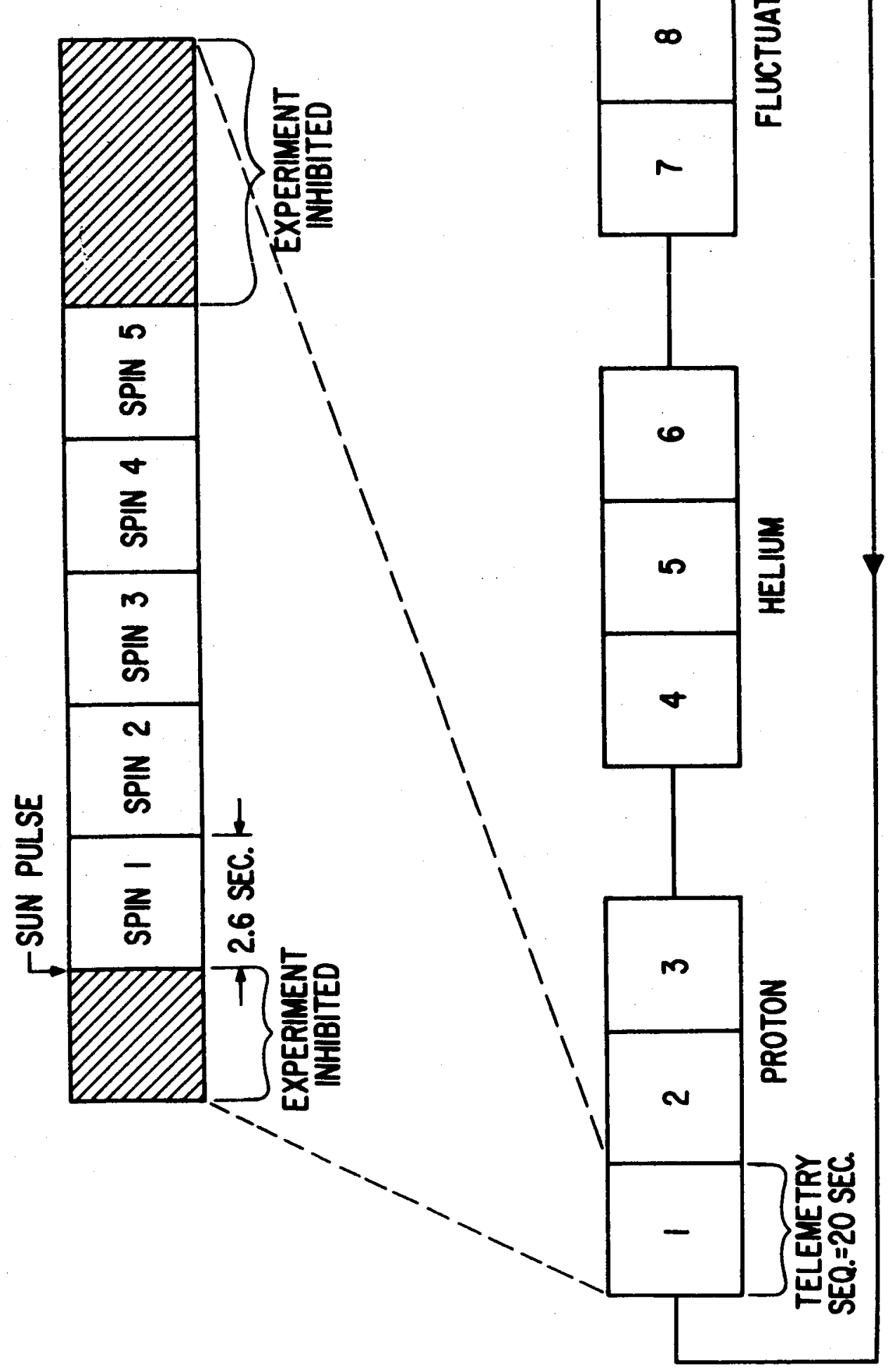

몰 


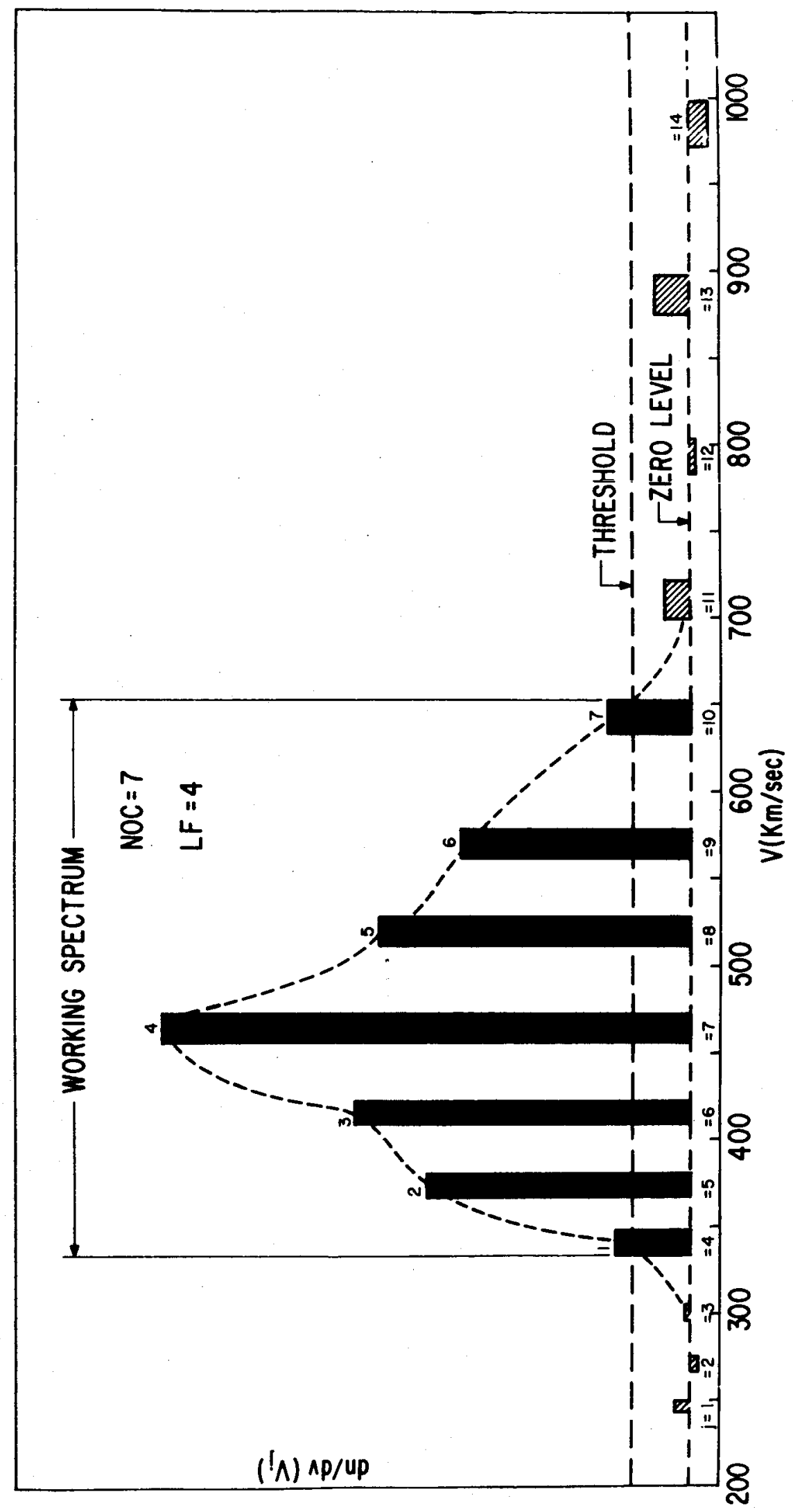



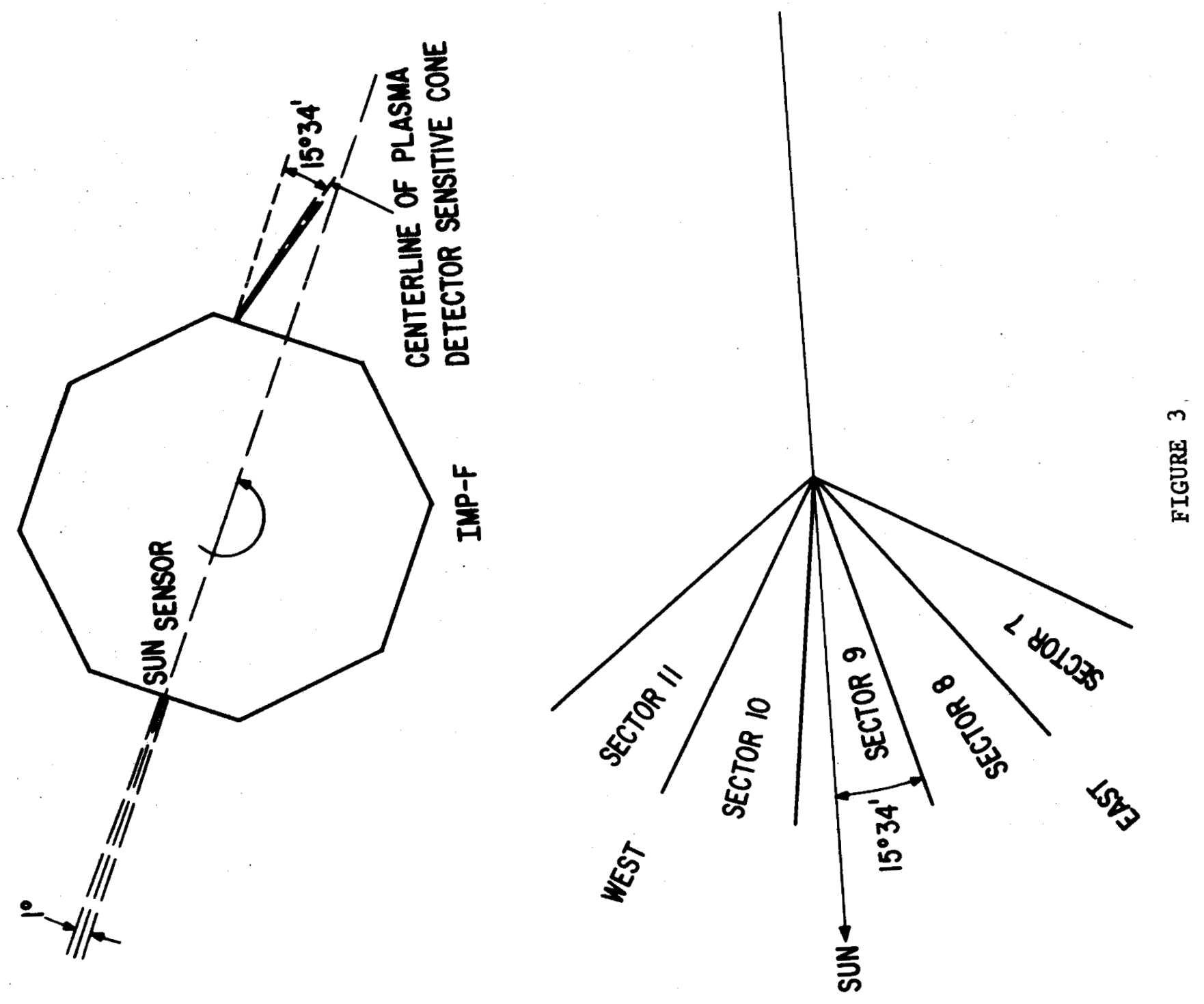


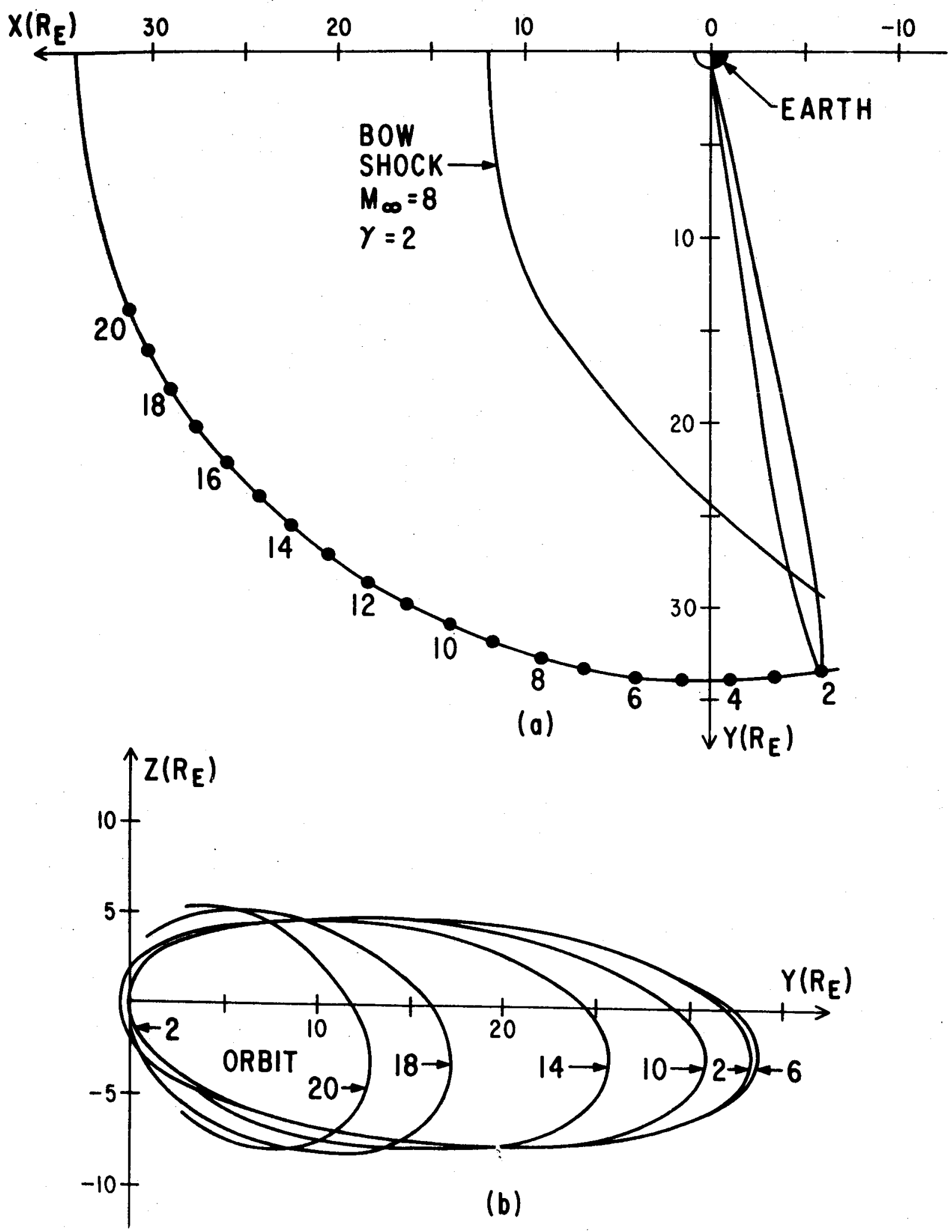




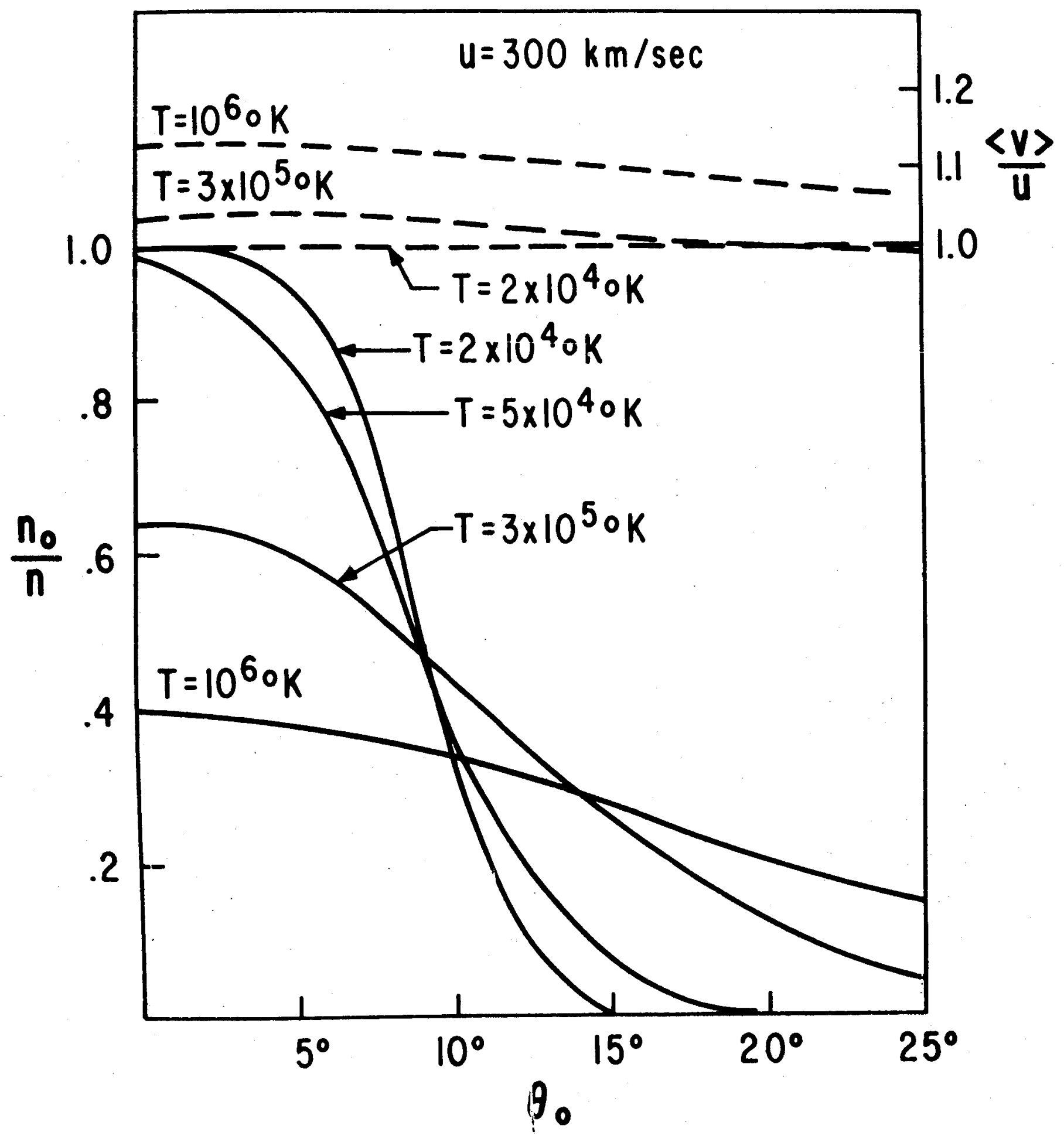

FIGURE 5 

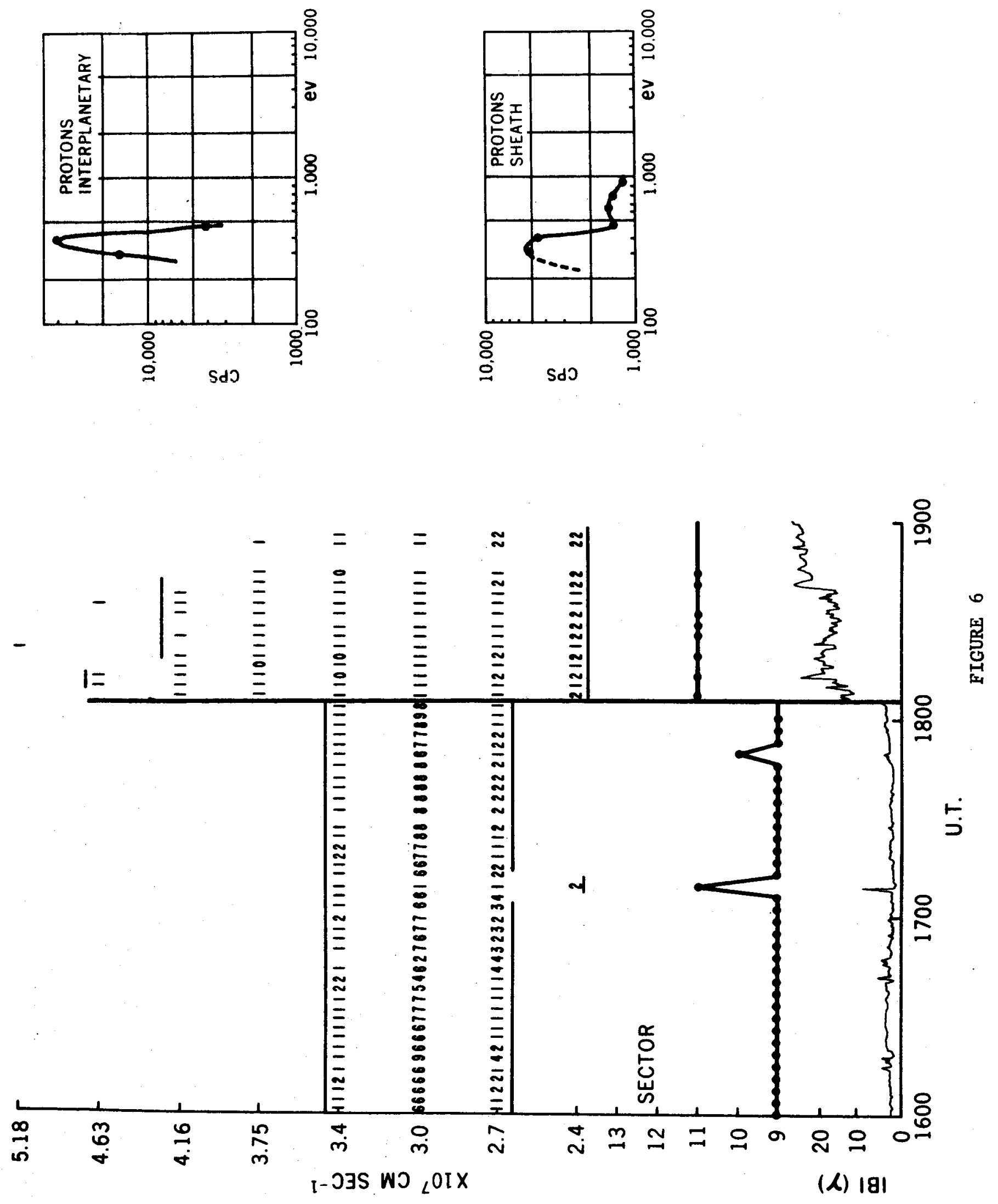


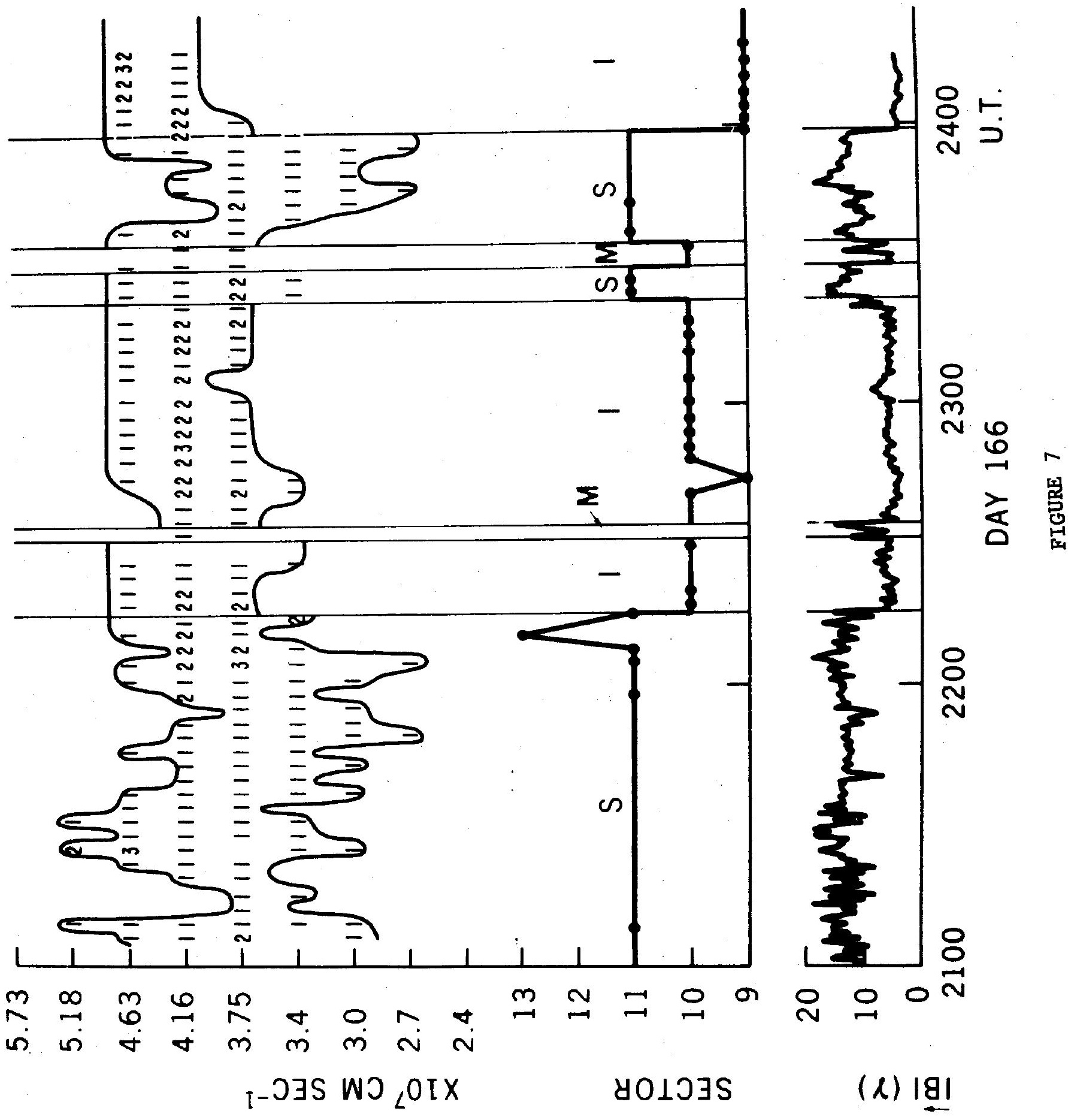




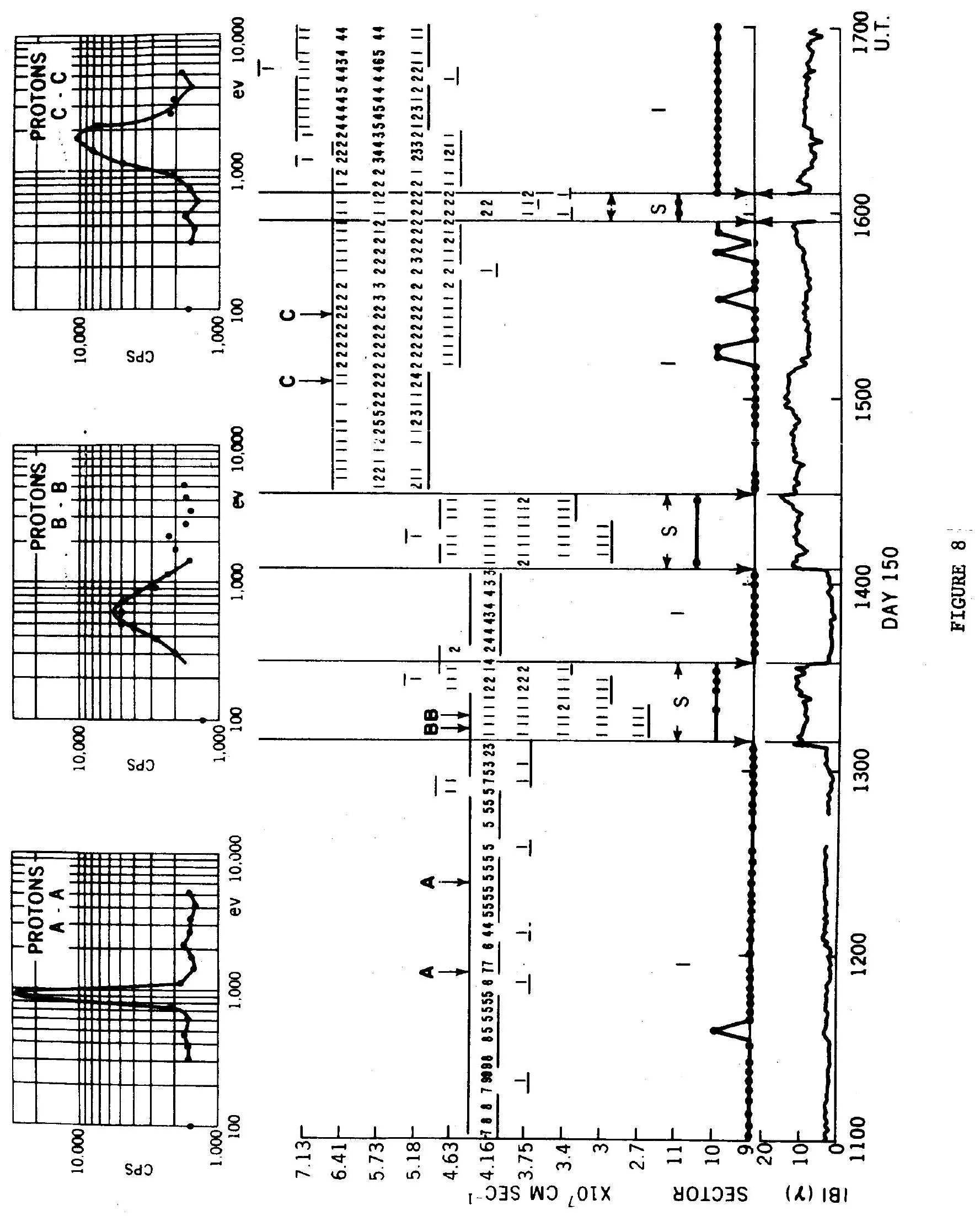




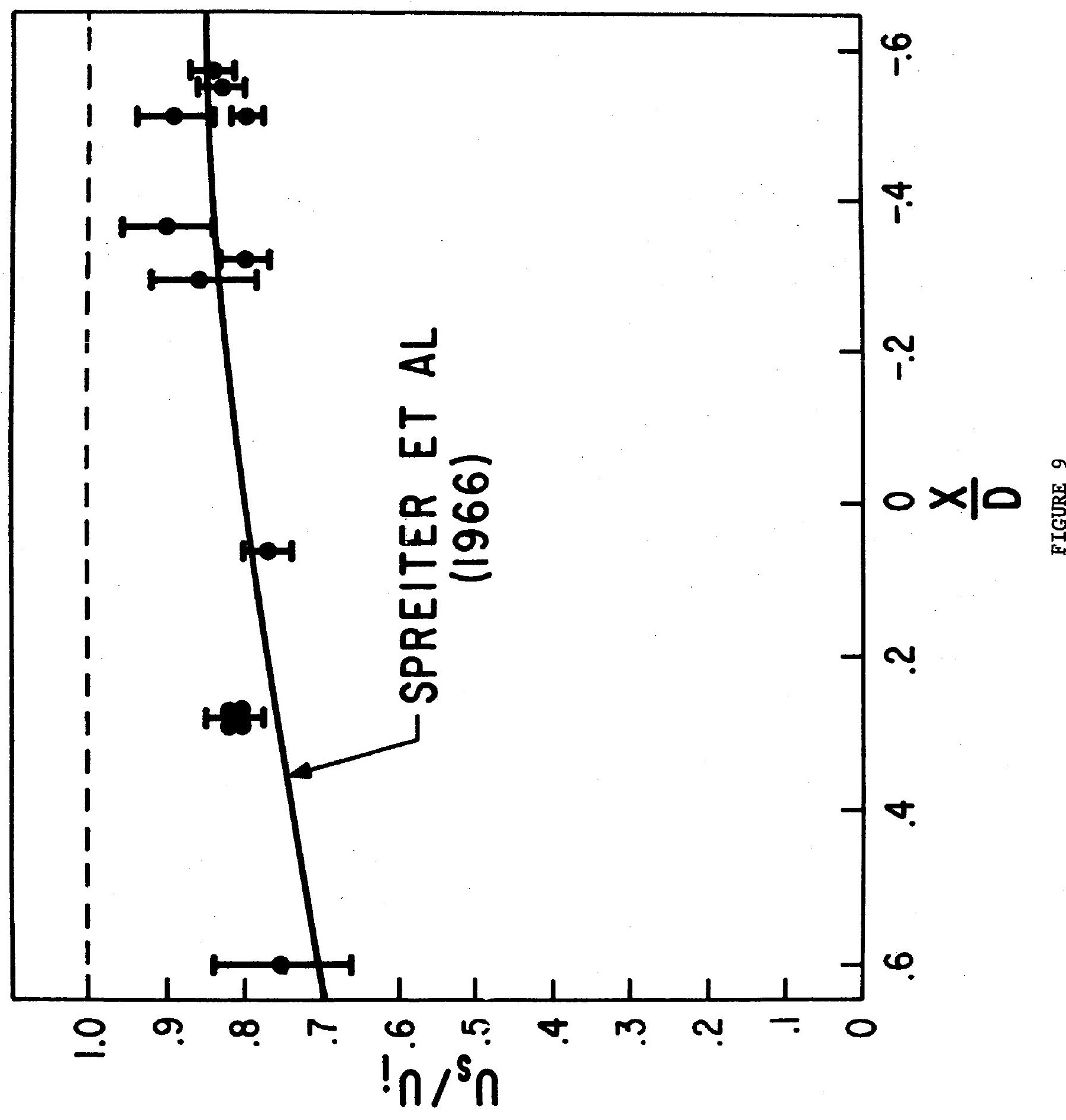




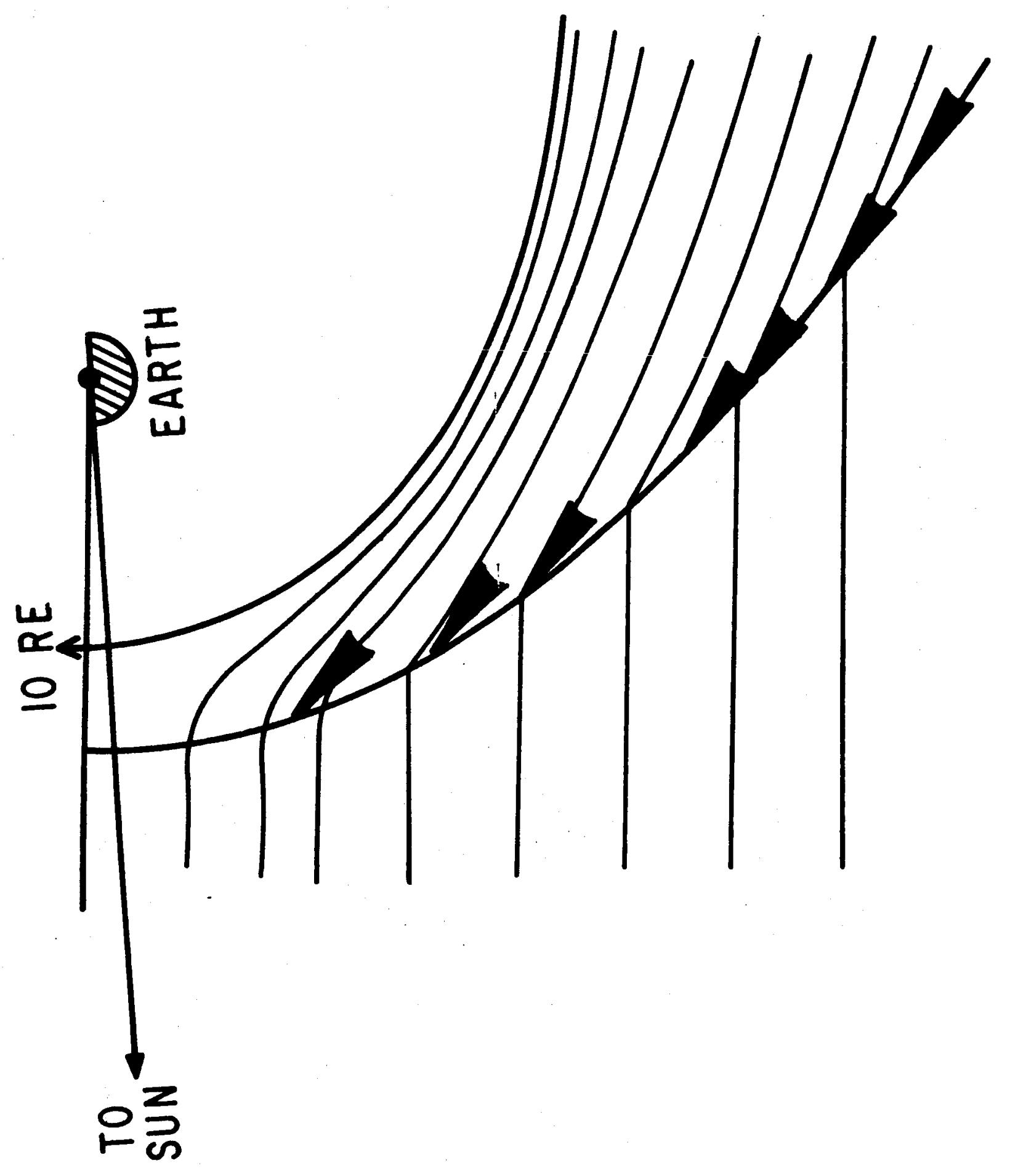

어
幽
芻
罗 


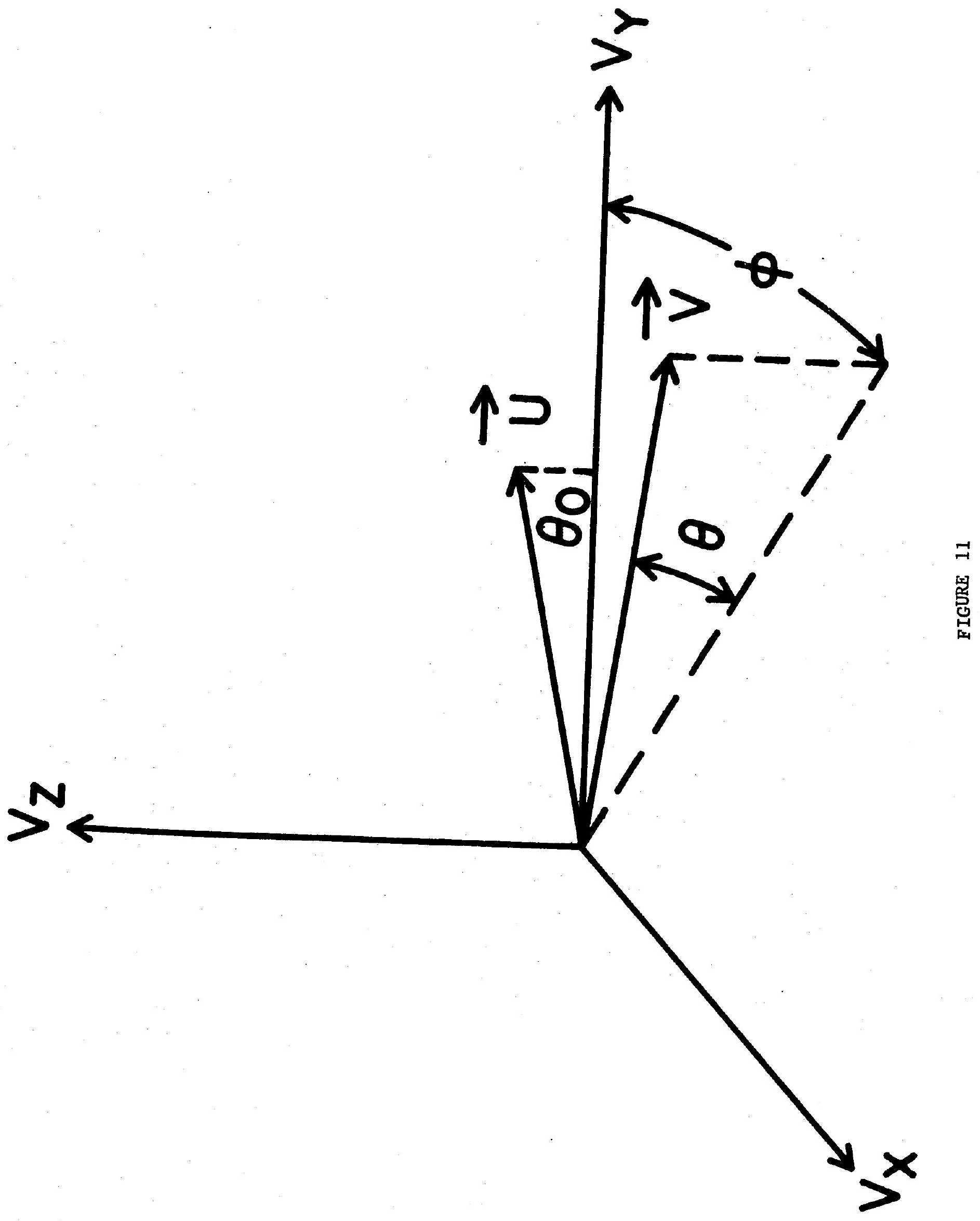

